\title{
2x2 Bismuth-Oxide-Fiber Based Crossbar Switch for All-Optical Switching Architectures
}

\author{
O. Zouraraki ${ }^{1}$, P. Bakopoulos ${ }^{1}$, K. Vyrsokinos ${ }^{1}$, and H. Avramopoulos ${ }^{1}$ \\ ${ }^{1}$ Photonics Communications Research Laboratory, National Technical University of \\ Athens, Zographou, GR 15773, Athens, Greece \\ ozour@mail.ntua.gr
}

\begin{abstract}
We demonstrate an optically controlled 2x2 Crossbar switch suitable for all-optical switching architectures. The switch is based on an Ultrafast Nonlinear Interferometer (UNI) configuration and uses 80 centimeters of highly nonlinear Bismuth Oxide fiber. Error-free operation is demonstrated with 10 and $40 \mathrm{~Gb} / \mathrm{s}$ input signals, with power penalties in its BAR and CROSS states of less than $0.3 \mathrm{~dB}$ and $1 \mathrm{~dB}$ respectively. The short length of the highly nonlinear Bismuth Oxide fiber ensures compact size, small latency and good operational stability of the switch.
\end{abstract}

Keywords: Optically controlled $2 \times 2$ switch, Bismuth Oxide Nonlinear fiber, Ultrafast Nonlinear Interferometer (UNI), all-optical signal processing.

\section{Introduction}

The increasing demand in ultrahigh capacity, global data networks has pushed intelligent switching functionalities to the optical layer. [1]. All-optical switching is believed to play a vital role in future broad-band communications systems as it uses capacity more efficiently, increases throughput, provides greater flexibility than pure wavelength-division multiplexing [2] and offers format and bitrate transparency of data [3]. Towards this end, optical switching has been investigated in a plethora of architectures that incorporate optical cross-connects for optical packet routing, timeslot interchangers for packet re-arranging and collision avoiding, optical add/drop multiplexers, optical buffers and optical signal monitoring schemes [2]. In most optical switch-based approaches, $2 \times 2$ and $1 \times 2$ switches are used as the core switching elements [3] since their appropriate cascading enables the implementation of switching subsystems that can accommodate more than two input signals. For example switches of size larger than $2 \times 2$ can be implemented using $2 \times 2$ switches as the basic building block. In the past, various efficient architectures for building large switches using fundamental switching elements have been presented [2][4]. Another application for cascaded $2 \times 2$ switches is also optical buffering schemes [5][6]. Such applications pose significant performance requirements on the $2 \times 2$ switching elements, such as switch bitrate scalability in order to increase the maximum link utilization, cascadability and high extinction ratio [1][7] whereas signal-to-noise ratio deterioration is especially crucial in feedback applications. 
So far, $2 \times 2$ all-optical switches have been associated with semiconductor-based devices [11][12]. Semiconductors have been considered more suitable for all-optical switching applications as they offer significant advantages including high nonlinearity, compact size and capability for integration. Nonlinear fibers, on the other hand, provide the unique advantages of fs nonlinearity response, nearly penaltyfree switching and operation without electrical power supply, but have traditionally required long, bulky fiber spans. However recent advances in the fabrication of highly-nonlinear fibers (HNLF) led to the construction of high quality nonlinear fibers which allow for extensive size reduction of fiber-based implementations. One of the most attractive candidate for fiber-based switching applications is Bismuth Oxide $\left(\mathrm{Bi}_{2} \mathrm{O}_{3}\right)$ based nonlinear fiber $(\mathrm{Bi}-\mathrm{NLF})$ that exhibits an extremely high nonlinearity exceeding $1000 \mathrm{~W}^{-1} \mathrm{~km}^{-1}$. This means that a meter or less [9][10] of this kind of fiber would be enough for the implementation of ultra-compact, all-optical fiber switches. Besides compact size, short nonlinear fiber lengths enhance operational stability and most significantly guarantee low switch latency. This could be very crucial when cascading a large number of switches, as well as in feedback applications such as feed-back buffering schemes.

In this letter we demonstrate an optically controlled $2 \times 2$ Crossbar switch using 80 centimeters of highly nonlinear Bi-NLF. The switch is based on a single-arm, Ultrafast Nonlinear Interferometer (UNI) gate [11], which uses the $80-\mathrm{cm}$ long BiNLF as the nonlinear interaction medium in order to obtain a complete $\pi$ phase shift. The below-200 fs nonlinearity response time of the highly nonlinear fiber [13] ensures that the switching window of the switch is defined by the width of the control pulses. This has enabled bitwise operation at input data rates of $40 \mathrm{~Gb} / \mathrm{s}$, whereas in principle switch operation can be extended at even higher rates. Error-free operation has been achieved with power penalties in its BAR and CROSS states of less than 0.3 $\mathrm{dB}$ and $1 \mathrm{~dB}$ respectively. The proposed $2 \times 2$ Crossbar switch can comprise the key unit for the implementation of a full range of $2 \times 2$ switch-based circuits including switching matrices [2][4], time-slot interchangers [14], header reinsertion circuits [15] and optical buffers [1] [16].

\section{Experimental Setup}

The $2 \times 2$ crossbar switch was tested to operate with both $10 \mathrm{~Gb} / \mathrm{s}$ and $40 \mathrm{~Gb} / \mathrm{s}$ input data signals as follows. A single $10 \mathrm{~Gb} / \mathrm{s}$ data channel along with a $40 \mathrm{~Gb} / \mathrm{s}$ timedivision-multiplexed (TDM) signal enter the fiber UNI, from inputs IN 1 and IN 2 respectively. In the absence of the control signal, the input signals exit the switch from OUT 1 and OUT 2 unchanged. Injection of the control signal causes the Cross function, namely one $10 \mathrm{~Gb} / \mathrm{s}$ channel of IN 2 is switched to OUT 1, whereas the input signal of IN 1 is switched to OUT2 to form the new $40 \mathrm{~Gb} / \mathrm{s}$ TDM output signal.

Fig. 1 shows the experimental setup used for the evaluation of the $2 \times 2$ crossbar switch. A $1553 \mathrm{~nm}$ DFB laser diode was gain switched at $10 \mathrm{GHz}$ to generate 10-ps pulses. These pulses were compressed to 3 ps in a nonlinear fiber pulse compressor and were then modulated with a $\mathrm{Ti}: \mathrm{LiNbO}_{3}$ modulator (MOD1) to form a pseudorandom data pattern. The signal was subsequently split in a $3 \mathrm{~dB}$ coupler into 
two parts: The first part entered an all-optical wavelength converter consisting of an integrated, SOA-based Mach Zehnder interferometer (MZI 1) to generate a $10 \mathrm{~Gb} / \mathrm{s}$ input signal (IN 1) to the $2 \times 2$ switch at $1558 \mathrm{~nm}$. The second part was rate quadrupled into a fiber bit interleaver, to produce a $40 \mathrm{~Gb} / \mathrm{s} 2^{7}-1$ PRBS data pattern (IN 2). The control signal was obtained by another $10-\mathrm{GHz}$ gain switched laser (DFB 2) operating at $1534 \mathrm{~nm}$ and producing 8.2 ps clock pulses. These pulses were modulated in a $\mathrm{Ti}: \mathrm{LiNbO}_{3}$ modulator (MOD2) to produce bursts of clock pulses with $2.3 \mathrm{nsec}$ duration and $40.5 \mathrm{nsec}$ period and were amplified in a high power EDFA.

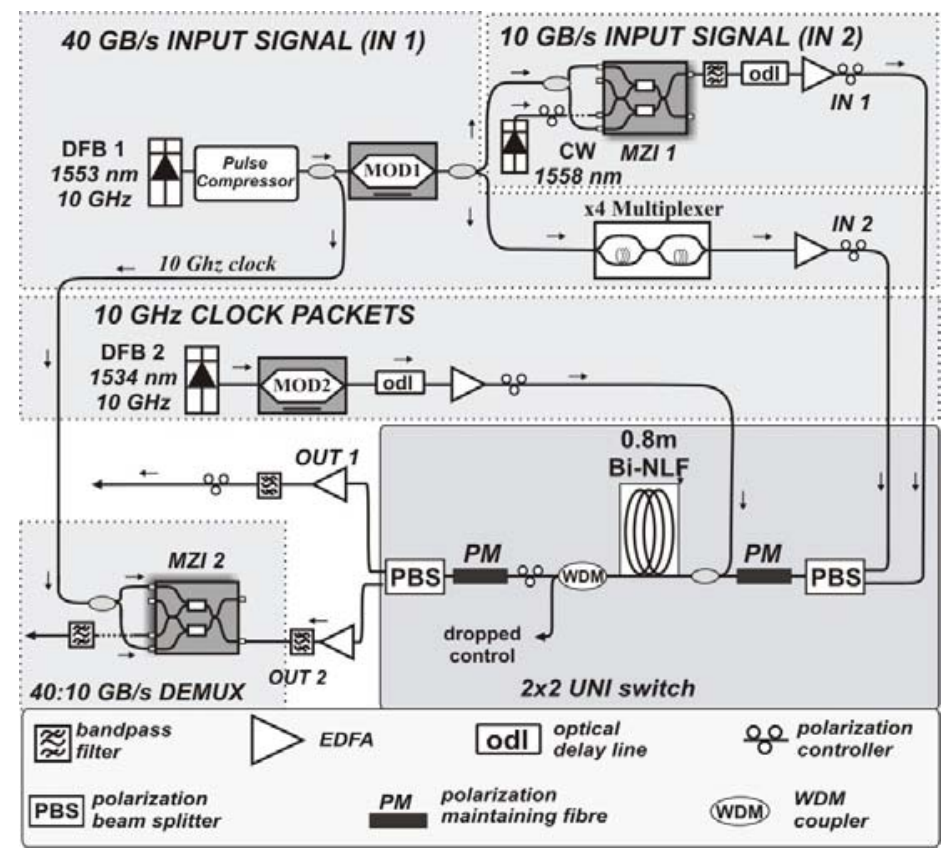

Fig. 1. Experimental setup

These three signals were injected into the $2 \times 2$ Crossbar switch which was implemented with a 2-input, 2-output UNI gate as shown in Fig. 1. The nonlinear element in the UNI was a 80-cm long, highly nonlinear $\left(\gamma=950 \mathrm{~W}^{-1} \mathrm{Km}^{-1}\right)$ Bi-NLF which was pigtailed with single mode fiber (SMF). Core diameter of the Bi-NLF was $2 \mu \mathrm{m}$. The Bi-NLF exhibited $1.3 \mathrm{~dB} / \mathrm{m}$ propagation losses whereas the input pigtail splice introduced an additional $2.1 \mathrm{~dB}$ loss. The time-synchronized input signals entered the switch in orthogonal polarizations from the ordinary and extraordinary ports of a Polarization Beam Splitter (PBS) respectively. A $45^{\mathrm{O}}$ splice analyzed each signal in two equal orthogonal components which are temporally separated by the polarization maintaining (PM) fiber section providing $10 \mathrm{ps}$ of birefringent delay. The control signal was launched into the switch through a 90:10 fiber coupler so that it cotravels with the input signals. Fine bitwise synchronization between the three optical signals was achieved with two optical delay lines, so that the control pulse coincided with the trailing component of both input signals. Re-alignment of each input signal 
component was achieved by another PM section of equal length where the fast/slow signal components were aligned to the slow/fast PM fiber axis respectively by means of a polarization controller. A $45^{\mathrm{O}}$ splice followed by a PBS perform spatial separation of the resultant signals at the corresponding PBS outputs according to their polarization state. At the output of the switch and in the absence of the control signal, data signal IN 1 passes through to OUT 1 and data signal IN 2 passes through to OUT 2. In the presence of the control signal, the polarization state of each input data component which temporally coincides with the control is rotated by $90^{\circ}$. As a result, the $10 \mathrm{~Gb} / \mathrm{s}$ channel of IN 1 and the synchronized $10 \mathrm{~Gb} / \mathrm{s}$ data channel of IN 2 are interchanged at the outputs of the switch. After interacting with the two input data signals in the nonlinear fiber, the control signal was removed with a $1550 / 1530 \mathrm{~nm}$ wavelength selective coupler. Finally, bit error rate (BER) measurements were performed at both outputs of the switch. The BER performance of the $40 \mathrm{~Gb} / \mathrm{s}$ signal of OUT 2 was assessed with a 40 -to-10-Gb/s data demultiplexer, so as to evaluate each of the $10 \mathrm{~Gb} / \mathrm{s}$ tributary channels. Demultiplexing was performed with a second integrated Mach Zehnder interferometer (MZI 2) operating in push-pull control configuration, which was controlled with 3 ps clock pulses at $1553 \mathrm{~nm}$.

\section{Results and Discussion}

The experimental results of the $2 \times 2$ Crossbar switch are illustrated in Figures 2, 3 and 4. Fig. 2 depicts the oscilloscope traces for the BAR state operation of the switch whereas Fig. 3 shows the corresponding traces for the CROSS state along with the respective control signal. The arrows shown in Fig. 3 indicate the time slots which coincide with the control pulses. $2 \times 2$ switching occurs throughout the entire time window shown in Fig. 3, which is shorter than the burst length. In the BAR state (Fig. 2), data signal IN 1 exits the switch from OUT 1 while data signal IN 2 passes through to OUT 2. In the CROSS state (Fig. 3), the $10 \mathrm{~Gb} / \mathrm{s}$ channel of IN 2 coinciding with the control pulses is switched to OUT 1, whereas the input signal of IN 1 is switched to OUT2 to form a new $40 \mathrm{~Gb} / \mathrm{s}$ output signal. Fig. 4 illustrates the input and output eye diagrams for both states of the switch as obtained at both output ports simultaneously. The eye diagrams demonstrate an extinction ratio of approximately $10.1 \mathrm{~dB}$ for OUT1 and $11 \mathrm{~dB}$ for OUT2 in BAR state operation. For the CROSS state, the extinction ratio was $9.5 \mathrm{~dB}$ for OUT1 and $10 \mathrm{~dB}$ for OUT2. 


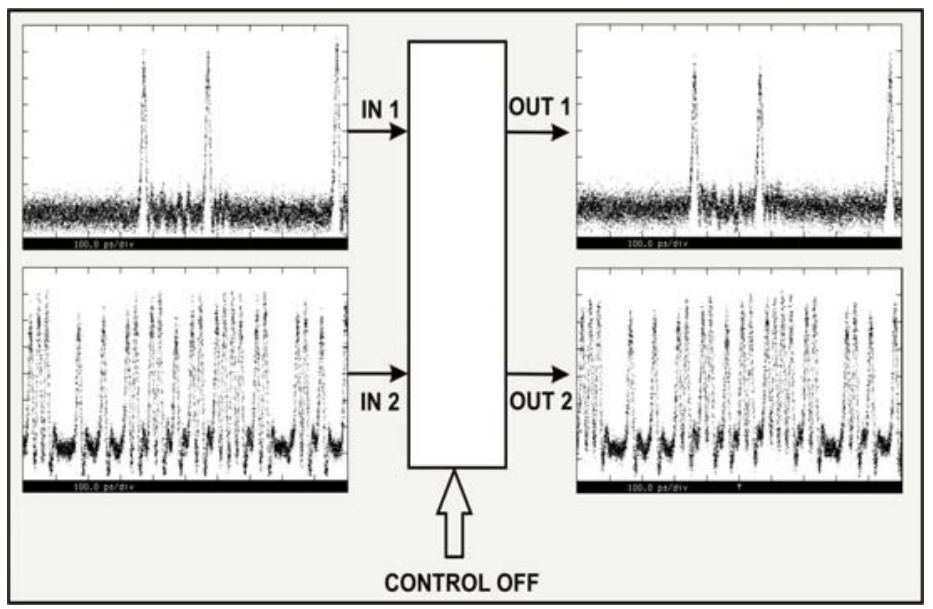

Fig. 2. Oscilloscope traces in the BAR state: The time base is $100 \mathrm{ps} / \mathrm{div}$

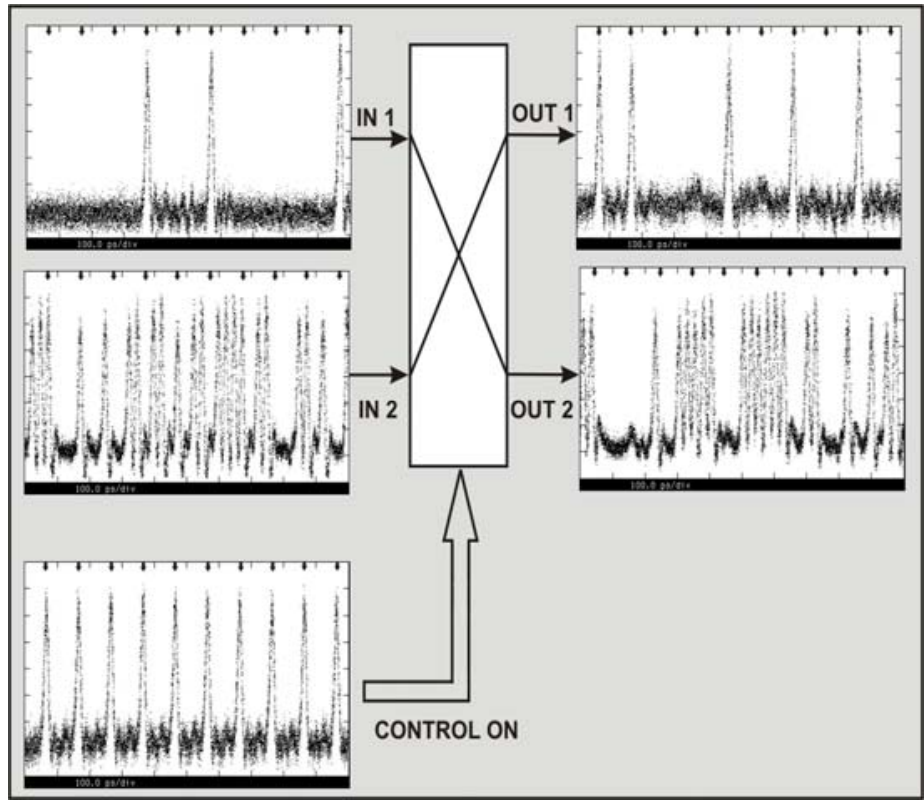

Fig. 3. Oscilloscope traces in the CROSS state: The time base is $100 \mathrm{ps} / \mathrm{div}$. Arrows indicate the time slots which coincide with the control pulses. 


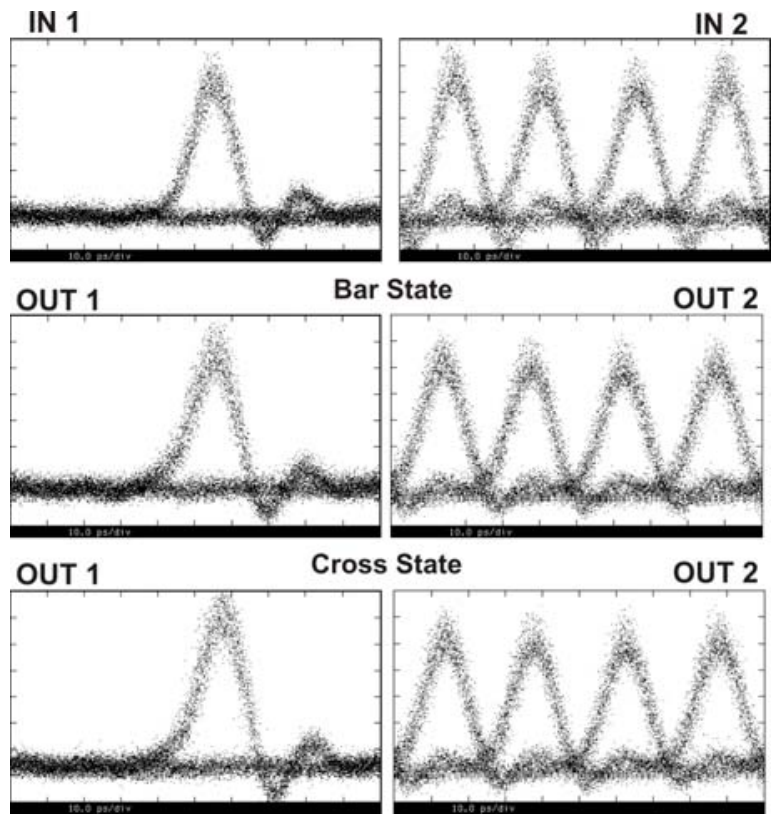

Fig. 4. Eye diagrams for the $2 \times 2$ Crossbar operation of the switch: First row: input signals, second row: BAR state operation, third row: CROSS state operation. (time scale: $10 \mathrm{ps} / \mathrm{div}$ ).

The Bit-Error-Rate (BER) performance of the switch was investigated with a 10 $\mathrm{Gb} / \mathrm{s}$ Bit Error Rate Tester and is illustrated in Fig. 5. BER curves are presented for the $10 \mathrm{~Gb} / \mathrm{s}$ signals of IN 1 and OUT 1 along with the corresponding $10 \mathrm{~Gb} / \mathrm{s}$ tributary data channels of IN 2 and OUT 2 for both BAR and CROSS states. Error-free $2 \times 2$ operation was achieved in both BAR and CROSS states with low power penalties. In BAR state operation, a power penalty of $0.3 \mathrm{~dB}$ and $0.28 \mathrm{~dB}$ was obtained for OUT 1 and OUT 2 ports respectively. CROSS state operation introduced a power penalty of $0.8 \mathrm{~dB}$ and $1.06 \mathrm{~dB}$ for OUT1 and OUT2 ports. For this operation the peak power of the control pulses was $8.5 \mathrm{~W}$, corresponding to $69.7 \mathrm{pJ}$ pulse energy and a complete $\pi$ nonlinear phase change. These power levels refer to the input of the Bi-NLF fiber and exclude the splice losses between the SMF pigtail and the Bi-NLF fiber. The polarization of both input signals was adjusted so as to maximize power transmitted through the input PBS, whereas control signal polarization was set with view to optimum switching. Once all polarizations were adjusted, the switch provided stable operation without the need for further polarization control, owing to the shortness of the Bi-NLF. The additional $2 \mathrm{~dB}$ power penalty at the input $10 \mathrm{~Gb} / \mathrm{s}$ signal (IN 1) compared to the input $40 \mathrm{~Gb} / \mathrm{s}$ (IN 2) is due to OSNR degradation in the wavelength conversion process in MZI 1. 
ㅁ IN $2(40 \mathrm{GHz}) \circ$ Bar State OUT $2(40 \mathrm{GHz}) \triangle$ Cross State OUT $2(40 \mathrm{GHz})$

- IN $1(10 \mathrm{GHz})$ - Bar State OUT $1(10 \mathrm{GHz})$ \& Cross State OUT $1(10 \mathrm{GHz})$

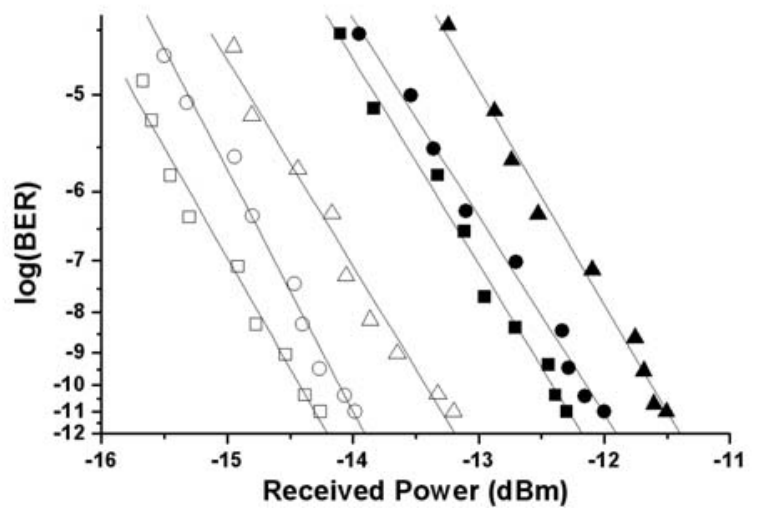

Fig. 5. BER measurements in BAR and CROSS states.

\section{Conclusion}

We have demonstrated an optically controlled $2 \times 2$ Crossbar switch using only 80 centimeters of highly nonlinear Bismuth Oxide fiber in a UNI configuration. The short length of the Bi-NLF fiber ensures to the switch compactness, enhances polarization stability and allows for small latency of the device. Error-free $2 \times 2$ operation was demonstrated with low power penalties for both $10 \mathrm{~Gb} / \mathrm{s}$ and $40 \mathrm{~Gb} / \mathrm{s}$ signals, indicating the switch may be cascaded or used in feedback applications, without requiring regeneration. The proposed $2 \times 2$ Crossbar switch can comprise the basic building block for the implementation of a full range of $2 \times 2$ switch-based circuits including large-scale switching subsystems, time-slot interchangers and optical buffers.

Acknowledgments. This work has been supported by the European Commission through project IST-ePhoton/One + (027497). The authors would like to thank Dr N. Sugimoto and ASAHI GLASS Company Ltd for providing the highly nonlinear BiNLF.

\section{References}

1. Emily F. Burmeister, John E. Bowers, "Integrated Gate Matrix Switch for Optical Packet Buffering”, IEEE Photon. Technol. Lett., vol. 18, issue 1, pp. 103-105, Jan. 2006.

2. G.I. Papadimitriou, C. Papazoglou, A.S. Pomportsis, "Optical switching: switch fabrics, techniques, and architectures", IEEE/OSA J. Lightwave Technol., vol. 21, issue 2, pp. 384405, Feb. 2003. 
3. D.K. Hunter, D.G. Smith, "New architectures for optical TDM switching", IEEE/OSA J. Lightwave Technol., vol. 11, issue 3, pp. 495 - 511, Mar. 1993.

4. 7. Rajiv Ramaswami, Kumar N. Sivarajan, "Optical Networks. A Practical Perspective", Sec-ond Edition, Academic Press, 2002.

5. D.K. Hunter, M.C. Chia, I. Andonovic, "Buffering in optical packet switches", IEEE/OSA J. Lightwave Technol., vol. 16, issue 12, pp. 2081 - 2094, Dec. 1998.

6. D.K. Hunter et al, " $2 \times 2$ buffered switch fabrics for traffic routing, merging, and shaping in photonic cell networks", IEEE/OSA J. Lightwave Technol., vol. 15, issue 1, pp. 86-101, Jan. 1997.

7. C.P. Larsen, M. Gustavsson, "Linear crosstalk in $4 \times 4$ semiconductor optical amplifier gate switch matrix”, IEEE/OSA J. Lightwave Technol., vol. 15, issue 10, pp. 1865 - 1870, Oct. 1997.

8. T. Hasegawa et al, "Bismuth-based extra-high nonlinear optical fiber", in Proc. Conference on Laser and Electro-Optics (CLEO), Baltimore, 2005, pp. 2094-2096, CFC2.

9. G. Meloni et al, "Ultrafast All-Optical ADD-DROP Multiplexer Based on 1-m-Long Bismuth Oxide-Based Highly Nonlinear Fiber", IEEE Photon. Technol. Lett., vol. 17, issue 12 , pp. 2661-2663, Dec. 2005.

10. J. H. Lee et al, "Wide-Band Tunable Wavelength Conversion of 10-Gb/s Nonreturn-to-Zero Signal Using Cross-Phase-Modulation-Induced Polarization Rotation in 1-m Bismuth Oxide-Based Nonlinear Optical Fiber", IEEE Photon. Technol. Lett., vol. 18, issue 1, pp. 298300, Jan. 2006.

11. G. Theophilopoulos, et al, "Optically Addressable 2x2 Exchange Bypass Packet Switch", IEEE Photon. Technol. Lett., vol. 14, issue 7, pp. 998-1000, Jul. 2002.

12. C.K. Yow, Y. J. Chai, C. W. Tee, R. McDougall, R. V. Penty, and I. H. White, "All-Optical Multiwavelength Bypass-Exchange Switching Using a Hybrid-Integrated Mach-Zehnder Switch", in Proc. Eur. Conf. Optical Communication, vol. 3, 2004, pp. 704-705, We4.P.118

13. N. Sugimoto, H. Kanbara, S. Fujiwara, K. Tanaka, Y. Shimizugawa, and K. Hirao, "Thirdorder optical nonlinearities and their ultrafast response in $\mathrm{Bi} 2 \mathrm{O} 3$ - B2O3 - SiO2 glasses ," J. Opt. Soc. Am. B 16, 1904-1908 (1999)

14. Varvarigos, E.M., "The "packing" and the "scheduling packet" switch architectures for almost all-optical lossless networks", IEEE/OSA J. Lightwave Technol., vol. 16, issue 10, pp. 1757-1767, Oct. 1998.

15. D. Tsiokos et al, "All-Optical $10 \mathrm{~Gb} / \mathrm{s}$ Header Replacement for Variable Length Data Packets”, in Proc. Eur. Conf. Optical Communication (ECOC), Rimini, Italy 2003, We4.P83.

16. S.Yao et al, "All-optical packet switching for metropolitan area networks: opportunities and challenges”, IEEE Communications Magazine, vol. 39, issue 3, pp. 142-148, Mar. 2001. 